\title{
Gente com quem, também, se faz a revista
}

\author{
Harley E. A. Bicas, Cristina Muccioli, Mauro S. O. Campos, Paulo E.C. Dantas, Mauro Goldchmit, \\ Samir J. Bechara, Vital Paulino Costa, Suzana Matayoshi
}

De entre os agradecimentos e homenagens devidas pelos Arquivos Brasileiros de Oftalmologia e que já se tornaram tradicionais, ao fim de cada ano de trabalhos, uma delas nos é especialmente importante trazer a conhecimentos gerais. A nomeação dos revisores dos trabalhos encaminhados à publicação é a forma singela de expressarmos a essas pessoas a gratidão da Oftalmologia brasileira pelas horas que lhe são graciosamente dedicadas a como ela vai se apresentar ao mundo, no presente e no futuro. De fato, a figura do analista de trabalhos numa revista científica identifica-se com a de seu maior patrimônio: a qualidade do que chega à luz, a garantia de confiabilidade no que se apresenta.

Ao apontar impropriedades de exposição e de conteúdo num artigo que se candidata à publicação, o conselheiro editorial beneficia um variado contingente de pessoas. Em primeiro lugar ao próprio autor, evitando-lhe a exposição de suas omissões e incorreções em matéria cuja publicação venha a ter recusada; ou permitindo-lhe seus aprimoramentos nas que vierem a ser aceitas, por seus aconselhamentos e sugestões. Em certos casos, a contribuição dada ao(s) autor(es) por um conselheiro editorial é, inequivocamente, maior que a de colaboradores do trabalho diretamente nomeados. Daí a razão de os Arquivos Brasileiros de Oftalmologia oferecerem aos analistas, ao fim do sigiloso processo de julgamento em que se aprova a publicação do artigo, a oportunidade de se declararem como seus examinadores.

Em segundo lugar, além da ajuda ao(s) autor(es), vem a prestada aos editores (facilitando-lhes as decisões sobre a publicação) e aos revisores da apresentação (linguagem, convenções biblioteconômicas). E, por fim, à comunidade científica, aos leitores em geral, provendo-lhes a substância da qual se nutre o conhecimento, renovando-lhe e expandindolhe os limites ao - de certo modo - avalizar as propostas de suas superações.

Essas responsabilidades e contribuições, cabentes aos conselheiros editoriais, não são exercidas, apenas, pelos que aparecem nomeados em cada um dos fascículos da revista, compondo um quadro que, embora numeroso, torna-se cada dia mais insuficiente para as necessidades das revisões. Neste ano que agora finda, por exemplo, foram recebidos até novembro $\mathbf{4 7 8}$ artigos para análise. Como pelo menos dois revisores são selecionados para cada matéria chega-se a um total de aproximadamente 956 exames, o que daria cerca de onze trabalhos por ano a cada um dos 68 titulados como conselheiros editoriais nacionais.
Bem vindos, pois, os "ad hoc". Vários, aliás, passam ao novo quadro dos "oficiais", em sua necessária renovação, pela presteza de suas respostas e pelo nível em que são dadas. Mas a todos, os mais e os menos acionados, nossas reverências em agradecimentos pelas contribuições. Neste 2007 são eles:
A. Duarte
Abelardo Couto Jr.
Acácio Alves de Souza Lima Filho
Adriana Berezovsky
Adriana Santos Forseto
Áisa Haidar Lani
Amélia Kamegasawa
Ana Estela Besteti Pires Ponce Sant'Anna
André Romano
André Messias
André Marcelo Vieira Gomes
Andrea Araújo Zin
Arnaldo Furman Bordon
Arnaud Araújo Filho
Belquiz Rodrigues A. Nassaralla
Breno Barth
Bruno Machado Fontes
Carlos Teixeira Brandt
Carlos Alexandre de Amorim Garcia
Carlos Eduardo Leite Arieta
Carlos Guillermo Arce
Celina Tamaki Monteiro de Castro
César Lipener
Christiane Rolim de Moura
Christiano Fausto Barsante
Cinthia Oyama
Claudio Renato Garcia
Cleusa Coral-Ghanem
Davi Araf
Denise Vuono Chinzon
Diane Ruschel Marinho
Eduardo Ferrari Marback
Elcio Hideo Sato
Elcio Roque Kleinpaul
Eliana Aparecida Forno
Enyr Saran Arcieri
Eric Pinheiro de Andrade
Erika Alessandra Silvino Rodrigues
Fabio Ejzenbaum
Fabio Henrique Cacho Casanova 
Fausto Uno

Felicio Aristóteles da Silva

Fernanda Bon Duarte

Flávio Rocha Lima Paranhos

Flávo Jaime Rocha

Francisco Assis Cordeiro Barbosa

Francisco Porfírio Neto Jr.

Geraldo de Barros Ribeiro

Helia Soares Angotti

Jackson Barreto Jr.

Jayter Silva de Paula

João Borges Fortes Filho

João Amaro Ferrari Silva

João Antonio Prata Junior

Joao Carlos Miranda Goncalves

João J. Nassaralla Jr

Joelice dos Santos Araújo

José Vital Filho

Keila Monteiro de Carvalho

Kimble Matos

Laurentino Biccas Neto

Lincoln Leme Freitas

Luciane B. Moreira

Luis Paves

Luis Antonio Vieira

Luis Carlos Ferreira de Sá

Luis Eduardo Morato Rebouças de Carvalho

Luiz Alberto Melo

Magno Antonio Ferreira

Maira Saad de Avila Morales

Marcelo Hatanaka

Marcelo Jordão Lopes da Silva

Marcelo Carvalho da Cunha

Marcelo Vieira Netto

Marcelo Francisco Gaal Vadas

Marcos Volpini

Marcos Carvalho da Cunha

Maria Aparecida Onuki Haddad

Maria de Lourdes Motta MoreiraVillas Boas

Maria Emilia Xavier dos Santos Araujo

Maria Kiyoko Oyamada

Maria Regina Catai Chalita

Maria Teresa Bonanomi

Mário Teruo Sato

Martha Maria Motono Chojniak

Mauricio Della Paolera
Mauricio Maia

Mauricio Bastos Pereira

Mauro Nishi

Mauro Waiswol

Midori Osaki

Milton Massato Hida

Mônica Fialho Cronemberger

Moysés Zajdenweber

Nelson Alexandre Sabrosa

Nilva Simeren B. Moraes

Niro Kasahara

Norma Allemann

Norma Helen Medina

Nubia Cristina Freitas Maia

Patricia Mitiko Santello Akaishi

Paulo Afonso Batista dos Santos

Raquel Rocha de Almeida Dantas

Renata Attanasio de Rezende

Ricardo Belfort

Ricardo Suzuki

Ricardo Themudo Lessa Waetge

Roberto Freda

Roberto Lauande Pimentel

Roberto Abucham

Roberto Pinto Coelho

Roberto Murad Vessani

Ronaldo Boaventura Barcellos

Rosa Maria Graziano

Rosana Nogueira Pires da Cunha

Rosane Cruz Ferreira

Rui Barroso Schimiti

Samuel Rymer

Sandra Maria Canelas Beer

Seiji Hayashi

Sérgio Luís Gianotti Pimentel

Sidney Julio de Faria e Sousa

Silvia Veitzman

Simone Finzi

Solange Rios Salomão

Tomás Fernando Scalamandre Mendonça

Vera Lúcia D. Monte Mascaro

Vinicius Coral Ghanem

Virgilio Centurion

Walter Yukihiko Takahashi

Wesley Ribeiro Campos

Zelia Maria da Silva Correa 